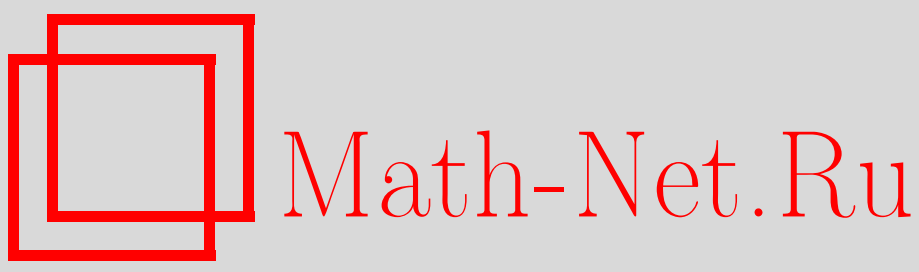

В. Ф. Кириченко, Л. И. Власова, Конциркулярная геометрия приближенно келеровых многообразий, Матем. сб., 2002, том 193, номер 5, 53-76

DOI: https://doi.org/10.4213/sm651

Использование Общероссийского математического портала Math-Net.Ru подразумевает, что вы прочитали и согласны с пользовательским соглашением http://www . mathnet.ru/rus/agreement

Параметры загрузки:

IP: 52.6 .47 .48

26 апреля 2023 г., 15:43:09 
УДК 514.76

В.Ф. Кириченко, Л.И. Власова

\title{
Конциркулярная геометрия приближенно келеровых многообразий
}

\begin{abstract}
В работе рассматриваются римановы многообразия, допускающие конциркулярные преобразования метрики. Исследуются конциркулярные инварианты почти эрмитовых многообразий. Подробно изучается геометрия почти эрмитовых многообразий, полученных конциркулярньм преобразованием метрики приближенно келеровых многообразий. Приведены новые примеры почти эрмитовых многообразий класса $W_{1} \oplus W_{4}$ постоянной кривизны как с интегрируемой, так и с неинтегрируемой структурой.

Библиографория: 31 название.
\end{abstract}

\section{§ 1. Введение}

Изучение конформно-инвариантных свойств римановых многообразий является одной из наиболее актуальных задач современной дифференциальной геометрии. В частности, традиционно важную роль в римановой геометрии играют (локально) конформно-плоские римановы многообразия [1]. Этот тип многообразий характеризуется тем, что (локально) конформным преобразованием метрики они переводятся в плоские римановы многообразия. В последние годы внимание исследователей все чаше привлекают локально конформно-келеровы многообразия, определяемые подобным образом [2], [3]. С другой стороны, А. Греем и Л. Эрвельей в работе [4] построена в известном смысле полная система основных классов почти эрмитовых многообразий и перечислены те классы, которые инвариантны относительно конформных преобразований метрики. Наиболее интересным из этих классов, на наш взгляд, является класс $W_{1} \oplus W_{4}$, обобшаюший класс $W_{1}$ приближенно келеровых многообразий и класс $W_{4}$, в размерности большей четырех совпадающий с классом локально конформно-келеровых многообразий. Именно эти классы наиболее интенсивно изучаются специалистами по эрмитовой геометрии. Существенный вклад в изучение приближенно келеровых многообразий внес А. Грей, а в изучение локально конформно-келеровых многообразий - И. Вайсман (см. библиографию). Поэтому многообразия класса $W_{1} \oplus W_{4}$ были названы многообразиями Вайсмана-Грея (короче, $V G$-многообразиями [5]). Среди $V G$-многообразий естественно выделяются локально конформно приближенно келеровы многообразия, образующие самостоятельньй конформно-инвариантный класс. Интересно отметить, что конформные преобразования метрики приближенно келерова многообразия выводят его за пределы класса приближенно келеровых многообразий [4]. $\mathrm{C}$ другой стороны, пока не известно ни одного примера $V G$-многообразий, отличных от локально конформно приближенно келеровых многообразий. 
Хорошо известно, что конформное преобразование метрики сохраняет геодезические (т.е. переводит геодезические в геодезические) тогда и только тогда, когда оно тривиально, т.е. является гомотетией. Существуют, однако, нетривиальные конформные преобразования, сохраняющие геодезические окружности (т.е. кривые, у которых первая кривизна постоянна, а остальные кривизны равны нулю). Они называются конциркулярными преобразованиями. Их изучение и является предметом настоящей статьи. Мы докажем, что метрика риманова многообразия допускает (нетривиальное) конциркулярное преобразование тогда и только тогда, когда это многообразие является пространством Фиалкова. Это позволило нам получить примеры римановых многообразий как допускающих конциркулярные преобразования метрики, так и не допускающих их. Доказано, что конциркулярные преобразования метрики сохраняют такие важные свойства, как эйнштейновость и (в случае почти эрмитовых многообразий) комплексная линейность тензора Риччи. Наиболее подробно изучены конциркулярно приближенно келеровы многообразия. Показано, что они удовлетворяют тождествам кривизны класса $R_{3}$ (т.е. имеют $J$-инвариантный тензор римановой кривизны) и $R_{2}$, причем они удовлетворяют тождеству $R_{1}$ (т.е. являются паракелеровыми) тогда и только тогда, когда они либо эрмитовы, либо шестимерны и имеют неинтегрируемую структуру, а их форма Ли удовлетворяет определенному дифференциальному уравнению. Получено исчерпывающее описание конциркулярно приближенно келеровых многообразий постоянного по Ванхекке типа. Показано, что конциркулярные преобразования метрики сохраняют свойство постоянства голоморфной секционной кривизны для почти эрмитовых многообразий. Это позволило дать исчерпывающее описание конциркулярно приближенно келеровых многообразий постоянной голоморфной секционной кривизны и построить примеры собственных конциркулярно приближенно келеровых многообразий постоянной кривизны как с интегрируемой, так и с неинтегрируемой структурой. Наконец, получено исчерпывающее описание конформно-плоских конциркулярно приближенно келеровых многообразий.

\section{§2. Конциркулярные преобразования метрики}

Пусть $\left(M^{n}, g=\langle\cdot, \cdot\rangle\right)$ - $n$-мерное псевдориманово многообразие размерности вьше двух; $\mathfrak{X}(M)$ - модуль гладких векторных полей на $M ; d$ - оператор внешнего дифференцирования; $\nabla$ - риманова связность метрики $g ; R, r, \kappa$-соответственно тензор Римана-Кристоффеля, тензор Риччи и скалярная кривизна метрики $g ; \delta-$ оператор кодифференцирования. Все многообразия, тензорные поля и подобные объекты предполагаются гладкими класса $C^{\infty}$.

Напомним, что переход от метрики $g$ к метрике $\bar{g}=e^{2 \sigma} g$, где $\sigma \in C^{\infty}(M)$, называется конформным преобразованием метрики. Функция $\sigma$ называется определяющей функиией. Если $\sigma=\mathrm{const}$, конформное преобразование называется гомотетией. В противном случае конформное преобразование называется нетривиальнылм. Основньп инвариантом конформного преобразования является тензор Вейля $\mathscr{C}$ конформной кривизны, определенный формулой

$$
\begin{aligned}
\mathscr{C}(X, Y) Z= & R(X, Y) Z+\frac{1}{n-2}\{\langle r(Y), Z\rangle X+\langle Y, Z\rangle r(X)-\langle r(X), Z\rangle Y \\
& -\langle X, Z\rangle r(Y)\}+\frac{\kappa}{(n-1)(n-2)}\{\langle X, Z\rangle Y-\langle Y, Z\rangle X\} .
\end{aligned}
$$


Обрашения в нуль тензора $\mathscr{C}$ необходимо и достаточно для того, чтобы многообразие $M^{n}$ при $n>3$ было конформно-плоским, т.е. метрика этого многообразия в некоторой окрестности произвольной его точки конформнњм преобразованием могла быть преобразована в плоскую метрику [1].

Как уже упоминалось, в классе конформных преобразований метрики естественно выделяются преобразования, сохраняющие геодезические окружности; это так называемые конциркулярные преобразования. Как показал Яно [6], они характеризуются тем, что их определяюшая функция является решением дифференциального уравнения (уравнения Яно)

$$
\sigma_{i, j}=\sigma_{i} \sigma_{j}+\beta g_{i j}
$$

где $\left\{\sigma_{i}\right\}$ - компоненты 1-формы $d \sigma,\left\{\sigma_{i, j}\right\}$ - компоненты ее ковариантного дифференциала, $\beta=\beta(\sigma) \in C^{\infty}(M)$.

Остановимся на вопросе существования конциркулярных преобразований. Пусть $\sigma$ - определяющая функция такого преобразования. Очевидно, уравнение Яно можно записать в безындексной форме

$$
\nabla d \sigma=d \sigma \otimes d \sigma+\beta g
$$

Пусть $\lambda \in C^{\infty}(M)$ - положительная функция. Рассмотрим 1-форму $\tau=\lambda d \sigma$. $\mathrm{C}$ учетом (2) имеем:

$$
\nabla \tau=d \lambda \otimes d \sigma+\lambda \nabla d \sigma=(d \lambda+\lambda d \sigma) \otimes d \sigma+\lambda \beta g
$$

Выберем $\lambda$ так, чтобы $d \lambda+\lambda d \sigma=0$, т.е. $d(\ln \lambda)=-d \sigma$. Достаточно положить $\ln \lambda=-\sigma$, т.е. $\lambda=e^{-\sigma}$. Тогда $\tau=e^{-\sigma} d \sigma=d\left(-e^{-\sigma}\right)$. В частности, $\tau-$ точная форма. При этом

$$
\nabla \tau=\gamma g
$$

где $\gamma=\lambda \beta$ - произвольная гладкая функция. Обратно, пусть 1-форма $\tau$ удовлетворяет уравнению (3). Из этого уравнения, в частности, следует, что $\nabla_{X}(\tau) Y-$ $\nabla_{Y}(\tau) X=0$, т.е. $d \tau=0$, а значит, форма $\tau$ локально точна. Следовательно, каждая точка $p \in M$ допускает окрестность $U_{p}$ такую, что $\left.\tau\right|_{U_{p}}=d f$, где $f \in C^{\infty}\left(U_{p}\right)$. Пусть $W \subset U_{p}$ - компактная окрестность точки $p$. Тогда $\left.f\right|_{W}$ - ограниченная функция, и поскольку она определена с точностью до константы, можно считать, что $\left.f\right|_{W}<0$, а значит, $f=-e^{-\sigma}$, где $\sigma=-\ln (-f)$. Отсюда $\tau=d\left(-e^{-\sigma}\right)=e^{-\sigma} d \sigma, d \sigma=e^{\sigma} \tau$, а значит, в силу (3) $\nabla d \sigma=d \sigma \otimes d \sigma+\beta g$, где $\beta=e^{\sigma} \gamma$. Следовательно, метрика на $M$ локально допускает конциркулярное преобразование. Это преобразование будет определено глобально тогда и только тогда, когда форма $\tau$ точна.

ОПРЕДЕЛЕНИЕ 1. Псевдориманово многообразие, на котором определена ненулевая 1-форма $\tau$, удовлетворяющая уравнению (3), называется пространством Фиалкова (в иной терминологии, эквидистантньм пространством).

Такие многообразия впервые были рассмотрены Фиалковьм [7]. Они играют существенную роль в теории геодезических отображений [8].

Итак, доказана 
ТЕОРема 1. Псевдориманово многообразие допускает нетривиальное локальное конциркулярное преобразование метрики тогда и только тогда, когда оно является пространством Фиалкова. Это преобразование определено глобально тогда и только тогда, когда форма $\tau$ точна.

СЛЕДСТВИЕ. Пространство Фиалкова с нулевым первым числом Бетти, в частности односвязное пространство Фиалкова, допускает глобально определенное конциркулярное преобразование метрики.

Типичньми примерами пространств Фиалкова являются пространства постоянной кривизны, а также субпроективные пространства [9].

С другой стороны, из (3) вытекает, что векторное поле $\xi$ на пространстве Фиалкова $M$, дуальное форме $\tau$, обладает свойством $\mathscr{L}_{\xi}(g)=2 \gamma g$ (где $\mathscr{L}$ - оператор дифференцирования Ли), а значит, является конформньм вектором Киллинга. Но тогда $M$ допускает локальную однопараметрическую групшу конформных диффеоморфизмов [10]. Если $M$ компактно, то векторное поле $\xi$ будет полньм, а соответствуюшие конформные диффеоморфизмы определены глобально. Предположим дополнительно, что $M$ неприводимо, а значит, $\nabla \xi \neq 0$. Тогда эти конформные диффеоморфизмы не могут быть изометриями. (В самом деле, в противном случае $\mathscr{L}_{\xi}(g)=0$, а значит, $\gamma=0$ и в силу (3) $\nabla \tau=0$, т.е. $\nabla \xi=0$.) Следовательно, связная компонента группы конформных преобразований многообразия $M$ не совпадает со связной компонентой его группы изометрий. С учетом известных результатов Голдберга и Кобаяси [11], а также Нагано [12] получаем

ПРЕДЛОЖЕНИЕ 1. Компактное однородное неприводимое пространство Фиалкова размерности выше трех изометрично сфере.

ПРЕДЛОЖЕНИЕ 2. Компактное неприводимое пространство Фиалкова с параллельным тензором Риччи размерности выше двух изометрично сфере.

Пример 1. Хорошо известно, что полное неприводимое шестимерное собственное приближенно келерово многообразие компактно и является многообразием Эйнштейна [13]. С учетом предложения 2 получаем, что такое многообразие является пространством Фиалкова (а значит, допускает нетривиальное конциркулярное преобразование метрики) тогда и только тогда, когда оно изометрично шестимерной сфере. В частности, однородные пространства $\mathrm{SO}(5, \mathbb{R}) / U(2)$ и $\mathrm{SU}(3) / S(U(1) \times U(1) \times U(1))$, снабженные канонической приближенно келеровой структурой [14], не допускают нетривиальных конциркулярных преобразований метрики.

Основным инвариантом конциркулярных преобразований является тензор $\mathscr{Z}$ типа $(3,1)$, определенный формулой

$$
\mathscr{Z}(X, Y) Z=R(X, Y) Z-\frac{\kappa}{n(n-1)}(\langle X, Z\rangle Y-\langle Y, Z\rangle X)
$$

и назьваемьй тензором конииркулярной кривизны [6]. Очевидно, этот тензор обладает всеми классическими свойствами симметрии тензора Римана-Кристоффеля. Как и в случае тензора Вейля, обрашения в нуль тензора $\mathscr{Z}$ необходимо и достаточно для того, чтобы многообразие было (локально) конциркулярно-плоским, 
т.е. метрика этого многообразия в некоторой окрестности произвольной его точки конциркулярньм преобразованием могла быть преобразована в плоскую метрику [6]. С другой стороны, из (4) видно, что обращение в нуль этого тензора равносильно постоянству кривизны многообразия, и, таким образом, конциркулярно-плоскими являются пространства постоянной кривизны и только они. В частности, многообразия постоянной кривизны допукают нетривиальные конциркулярные преобразования метрики и, значит, являются пространствами Фиалкова, о чем уже упоминалось.

Заметим, что тензор $\mathscr{C}$ также, в частности, является инвариантом конциркулярных преобразований, а значит, инвариантом является и тензор $\mathscr{E}=\mathscr{C}-\mathscr{Z}$. Назовем его вторым конииркулярным инвариантом. Найдем геометрический смысл обрашения в нуль этого инварианта. Заметим, что в силу (1) и (4) ковариантные компоненты тензоров $\mathscr{C}$ и $\mathscr{Z}$ вычисляются соответственно по формулам

$$
\begin{aligned}
\mathscr{C}_{i j k l}= & R_{i j k l}+\frac{1}{n-2}\left(r_{i k} g_{j l}+r_{j l} g_{i k}-r_{i l} g_{j k}-r_{j k} g_{i l}\right) \\
& +\frac{\kappa}{(n-1)(n-2)}\left(g_{j k} g_{i l}-g_{j l} g_{i k}\right) \\
\mathscr{Z}_{i j k l}= & R_{i j k l}-\frac{\kappa}{n(n-1)}\left(g_{j k} g_{i l}-g_{j l} g_{i k}\right) .
\end{aligned}
$$

Следовательно, компоненты тензора $\mathscr{E}$ вычисляются по формулам

$$
\mathscr{E}_{i j k l}=\frac{1}{n-2}\left(r_{i k} g_{j l}+r_{j l} g_{i k}-r_{i l} g_{j k}-r_{j k} g_{i l}\right)+\frac{2 \kappa}{n(n-2)}\left(g_{j k} g_{i l}-g_{j l} g_{i k}\right)
$$

Пусть $\mathscr{E}=0$. Свертывая правую часть (6) с тензором $g^{j l}$, в силу условия $n>2$ получаем, что

$$
r_{i k}=\frac{\kappa}{n} g_{i k}
$$

и, таким образом, $M$-многообразие Эйнштейна. Обратно, если $M$ - многообразие Эйнштейна, то, подставляя (7) в (6), получаем, что $\mathscr{E}_{i j k l}=0$, а значит, $\mathscr{E}=0$. Таким образом, доказана

ТЕОРЕМА 2. Обращение в нуль второго конииркулярного инварианта многообразия равносильно эйнштейновости этого многообразия.

СлЕДСТВИЕ. Эйнштейновость многообразия является конииркулярно-инвариантным свойством.

\section{§ 3. Почти эрмитовы многообразия}

Пусть $M$ - почти эрмитово (короче, $A \mathscr{H}$-) многообразие с $A \mathscr{H}$-структурой $\left.(J, g=\langle\cdot, \cdot\rangle), J^{2}=-\mathrm{id},\langle J X, J Y\rangle=\langle X, Y\rangle, \operatorname{dim} M=n=2 m\right\rangle 2, \Omega(X, Y)=$ $\langle X, J Y\rangle$ - фундаментальная форма структуры. Хорошо известно, что задание почти эрмитовой структуры на $M$ равносильно заданию $G$-структуры на $M$ со структурной группой $U(n)$, элементами пространства которой являются реперы, состоящие из попарно сопряженных собственных векторов структурного эндоморфизма $J$ и унитарные в естественной эрмитовой метрике комплексификации соответствующего касательного пространства (такие реперы называются $A$-реперамu) [13]. Эта $G$-структура называется присоединенной. Будем предполагать, что 
индексы $i, j, k, \ldots$ пробегают значения от 1 до $2 m$, а индексы $a, b, c, d, f, g, h$ - значения от 1 до $m$. Положим $\widehat{a}=a+m$. Как обычно, по индексам, заключенньм в квадратные (соответственно круглые) скобки, подразумевается альтернирование (соответственно симметризация). Легко проверить, что на пространстве присоединенной $G$-структуры компоненты тензоров $g$ и $J$ задаются матрицами

$$
\left(g_{i j}\right)=\left(\begin{array}{cc}
0 & I_{m} \\
I_{m} & 0
\end{array}\right), \quad\left(J_{j}^{i}\right)=\left(\begin{array}{cc}
\sqrt{-1} I_{m} & 0 \\
0 & -\sqrt{-1} I_{m}
\end{array}\right),
$$

где $I_{m}$ - единичная матрица порядка $m$. Поскольку $J$ и $g$ - тензоры типов $(1,1)$ и $(2,0)$ соответственно, их компоненты на пространстве расслоения всех комплексных реперов над $M$ удовлетворяют уравнениям

$$
\begin{aligned}
d J_{j}^{i}+J_{k}^{i} \omega_{j}^{k}-J_{j}^{k} \omega_{k}^{i} & =J_{j, k}^{i} \omega^{k} \\
d g_{i j}+g_{k j} \omega_{i}^{k}+g_{i k} \omega_{j}^{k} & =g_{i j, k} \omega^{k}
\end{aligned}
$$

где $\left\{\omega^{i}\right\},\left\{\omega_{j}^{i}\right\}$ - компоненты форм смещения и римановой связности $\nabla$ соответственно, $J_{j, k}^{i}, g_{i j, k}$ - компоненты ковариантного дифференциала тензоров $J$ и $g$ в этой связности соответственно. Более того, в силу определения римановой связности $\nabla g=0$ и, значит,

$$
g_{i j, k}=0 \text {. }
$$

С учетом (8) и (10) соотношения $(9)$ на пространстве присоединенной $G$-структуры перепишутся в форме

$$
\begin{gathered}
J_{b, k}^{a}=0, \quad J_{\widehat{b}, k}^{\widehat{a}}=0, \quad \omega_{\widehat{b}}^{\widehat{a}}+\omega_{a}^{b}=0, \\
\omega_{\widehat{b}}^{a}=-\frac{1}{2} \sqrt{-1} J_{\widehat{b}, k}^{a} \omega^{k}, \quad \omega_{b}^{\widehat{a}}=\frac{1}{2} \sqrt{-1} J_{b, k}^{\widehat{a}} \omega^{k} .
\end{gathered}
$$

Кроме того, заметим, что в силу вещественности соответствующих форм и тензоров $\bar{\omega}^{i}=\omega^{\widehat{\imath}}, \bar{\omega}_{j}^{i}=\omega_{\widehat{\jmath}}^{\widehat{\imath}}, \overline{\nabla J}_{j, k}^{i}=\nabla J_{\widehat{\jmath}, \widehat{k}}^{\widehat{\imath}}$, где $t \rightarrow \bar{t}$ - оператор комплексного сопряжения.

С учетом этих соотношений первая группа структурных уравнений римановой связности

$$
d \omega^{i}=\omega_{j}^{i} \wedge \omega^{j}
$$

на пространстве присоединенной $G$-структуры почти эрмитова многообразия запишется в следуюшей форме, называемой первой группой структурных уравнений АН̈-многообразия:

$$
\begin{aligned}
& d \omega^{a}=\omega_{b}^{a} \wedge \omega^{b}+B^{a b}{ }_{c} \omega^{c} \wedge \omega_{b}+B^{a b c} \omega_{b} \wedge \omega_{c}, \\
& d \omega_{a}=-\omega_{a}^{b} \wedge \omega_{b}+B_{a b}{ }^{c} \omega_{c} \wedge \omega^{b}+B_{a b c} \omega^{b} \wedge \omega^{c},
\end{aligned}
$$

где $\omega_{i}=g_{i j} \omega^{j}$,

$$
\begin{aligned}
B^{a b c}=\frac{\sqrt{-1}}{2} J^{a}{ }_{[\widehat{b}, \widehat{c}]}, & B_{a b c}=-\frac{\sqrt{-1}}{2} J^{\widehat{a}_{[b, c]}}, \\
B^{a b}{ }_{c}=-\frac{\sqrt{-1}}{2} J^{a}{ }_{\widehat{b}, c}, & B_{a b}{ }^{c}=\frac{\sqrt{-1}}{2} J^{\widehat{a}}{ }_{b, \widehat{c}} .
\end{aligned}
$$


При этом

$$
\omega_{\widehat{b}}^{a}=B^{a b}{ }_{c} \omega^{c}-\widetilde{B}^{a b c} \omega_{c}, \quad \omega_{b}^{\widehat{a}}=B_{a b}{ }^{c} \omega_{c}-\widetilde{B}_{a b c} \omega^{c},
$$

где $\widetilde{B}^{a b c}=\frac{1}{2} \sqrt{-1} J^{a}{ }_{\widehat{b}, \widehat{c}}, \widetilde{B}_{a b c}=-\frac{1}{2} \sqrt{-1} J^{\widehat{a}}{ }_{b, c}$, и

$$
\overline{B^{a b c}}=B_{a b c}, \quad \overline{B_{c}^{a b}}=B_{a b}^{c}, \quad \overline{\omega_{b}^{a}}=-\omega_{a}^{b} .
$$

Почти эрмитова структура в сравнении с псевдоримановой структурой допускает гораздо большее число инвариантов конформного преобразования метрики. Например, наряду с основным конформным инвариантом $A \mathscr{H}$-структуры - тензором $\mathscr{C}$ - ее конформными инвариантами являются также элементы спектра этого тензора. Напомним [15], [16], что всякий тензор $T$ типа $(r, 1)$ на $A \mathscr{H}$-многообразии $M$, рассматриваемый как $r$-линейное отображение

$$
T: \underbrace{\mathfrak{X}(M) \times \cdots \times \mathfrak{X}(M)}_{r \text { раз }} \rightarrow \mathfrak{X}(M),
$$

однозначно распадается в сумму тензоров того же вида, $\mathbb{C}$-линейных либо $\mathbb{C}$-антилинейных по своим аргументам. Совокупность таких тензоров называется $\mathrm{cne \kappa m-}$ ром тензора $T$. Число элементов спектра равно, очевидно, $2^{r}$, причем элементы спектра обозначаются символами $\underset{(k)}{T}$, где $k$ - десятичное число, двоичная запись которого в $s$-м разряде имеет нуль либо единицу в зависимости от того, является ли тензор $\underset{(k)}{T} \mathbb{C}$-линейным либо $\mathbb{C}$-антилинейным по $s$-му аргументу соответственно. В явном виде тензор $T$ задается соотношением

$$
\underset{(k)}{T}\left(X_{1}, \ldots, X_{r}\right)=\pi \circ T\left(\theta X_{1}, \ldots, \theta X_{r}\right)+\bar{\pi} \circ T\left(\bar{\theta} X_{1}, \ldots, \bar{\theta} X_{r}\right)
$$

где $\theta X_{s}=\pi X_{s}$, если в двоичной записи числа $k$ в $s$-м разряде стоит нуль, и $\theta X_{s}=\bar{\pi} X_{s}$, если в двоичной записи числа $k$ в $s$-м разряде стоит единица. Здесь $\pi=\frac{1}{2}(\mathrm{id}-\sqrt{-1} J), \bar{\pi}=\frac{1}{2}(\mathrm{id}+\sqrt{-1} J)$ - естественные проекторы на собственные подмодули эндоморфизма $J$, отвечающие собственньм значениям $\sqrt{-1}$ и $-\sqrt{-1}$ соответственно. Например, легко проверить, что спектр тензора $\nabla J$ почти эрмитова многообразия имеет вид:

$$
\underset{(0)}{\nabla J}=\underset{(2)}{\nabla J}=0, \quad \underset{(1)}{\nabla J}=-J \circ B, \quad \underset{(3)}{\nabla J}=J \circ C,
$$

где

$$
\begin{aligned}
& B(X, Y)=\frac{1}{2}\left(\nabla_{J X}(J) Y-\nabla_{X}(J)(J Y)\right), \\
& C(X, Y)=\frac{1}{2}\left(\nabla_{J X}(J) Y+\nabla_{X}(J)(J Y)\right)
\end{aligned}
$$

- (модифицированные) виртуальный и структурный тензоры $A \mathscr{H}$-многообразия соответственно. 
Изучение классов $A \mathscr{H}$-многообразий, характеризуемых обращением в нуль тех или иных конформных инвариантов, приводит к ряду интересных результатов [17]. То же самое можно сказать и об элементах спектра тензора $\mathscr{E}$, являющихся инвариантами конциркулярного преобразования метрики $A \mathscr{H}$-многообразия. Например, в силу (6) элемент $\mathscr{E}$ спектра тензора $\mathscr{E}$ на пространстве присоединенной $G$-структуры определяется компонентами

$$
\mathscr{E}_{b c d}=\frac{1}{m-1} r_{b[d} \delta_{c]}^{a}
$$

Поэтому соотношение $\underset{\mathscr{E}}{\mathscr{E}}=0$ равносильно соотношению $r_{b d} \delta_{c}^{a}-r_{b c} \delta_{d}^{a}=0$. Свертывая это соотношение по индексам $a$ и $c$, получаем, что $r_{b d}=0$, что, как легко видеть, равносильно комплексной линейности эндоморфизма Риччи, т.е. соотношению

$$
r \circ J-J \circ r=0 .
$$

Очевидно, верно и обратное, и, таким образом, справедливо

ПРЕДЛОЖЕНИЕ 3. Обращение в нуль конциркулярного инварианта $\mathscr{E}$ почти әрмитова многообразия равносильно комплексной линейности эндоморфизма Риччи этого многообразия.

СЛЕДСТВИЕ. Комплексная линейность эндоморфизма Риччи соответствующего почти эрмитова многообразия является конциркулярно-инвариантным.м свойством этого многообразия.

ЗАмечАнИЕ 1. Интересно отметить, что свойство комплексной антилинейности эндоморфизма Риччи почти эрмитова многообразия, вообше говоря, не является конциркулярно-инвариантным свойством. Именно, рассуждая аналогично, убеждаемся, что обращение в нуль остальных, вообще говоря, ненулевых конциркулярных инвариантов - элементов спектра $\mathscr{E}, \mathscr{E}$ и $\mathscr{E}$ - равносильно тождеству

$$
\text { (1) (2) (4) }
$$

$$
r \circ J+J \circ r=\frac{\kappa}{m} J
$$

справедливость которого на соответствующем почти эрмитовом многообразии является конциркулярно-инвариантным свойством этого многообразия.

Далее, как показали А. Грей и Л. Эрвелья [4], на почти эрмитовом многообразии $M$ внутренним образом определен еще один конформный инвариант - тензор $\mu$ типа $(2,1)$, заданньй формулой

$$
\mu(X, Y)=\nabla_{X}(J) Y+\frac{1}{2(m-1)}\{\langle\xi, Y\rangle X+\langle\xi, J Y\rangle J X-\langle X, Y\rangle \xi-\langle X, J Y\rangle J \xi\}
$$

где $\xi$ - вектор, дуальный форме $\delta \Omega$. В терминах этого тензора (и элементов его спектра) можно определить всеклассы $A \mathscr{H}$-многообразий (в классификации ГреяЭрвельи), инвариантные относительно конформных преобразований метрики. Например, тождеством $\mu(X, Y)=0$ характеризуется класс $W_{4}$, в размерности большей четырех совпадающий с классом локально конформно-келеровых многообразий [4]. В контексте нашей работы нас будут интересовать следующие два класса. 
ОПРЕДЕЛЕНИЕ 2. Почти эрмитово многообразие назьвается $G_{1}$-многообразиeм, если оно принадлежит классу $W_{1} \oplus W_{3} \oplus W_{4}$ в классификации Грея-Эрвельи [4].

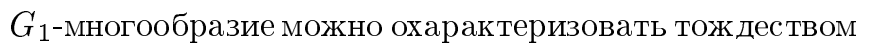

$$
\mu(X, X)=0
$$

(3)

или, эквивалентно,

$$
\mu(X, X)-\mu(J X, J X)=0 .
$$

Поляризуя это тождество, с учетом определения тензора $\mu$ получаем:

$$
\nabla_{X}(J) Y+\nabla_{Y}(J) X-\nabla_{J X}(J)(J Y)-\nabla_{J Y}(J)(J X)=0 .
$$

Покомпонентно расписьвая это тождество на пространстве присоединенной $G$-структуры, убеждаемся, что оно равносильно соотношениям

$$
B^{a b c}=B^{[a b c]} \text {. }
$$

Как известно, это равносильно антикоммутативности присоединенной $Q$-алгебры многообразия [15]. Стандартная процедура дифференциального продолжения соотношений (12) приводит ко второй группе структурных уравнений $G_{1}$-структуры:

$$
\begin{aligned}
d \omega_{b}^{a}= & \omega_{c}^{a} \wedge \omega_{b}^{c}+\left(2 B^{a d h} B_{h b c}+A_{b c}^{a d}\right) \omega^{c} \wedge \omega_{d} \\
& +\left(B^{a d}{ }_{[c} B_{d] b h}+A_{b c d}^{a}\right) \omega^{c} \wedge \omega^{d}+\left(-B_{b h}{ }^{[c} B^{d] a h}+A_{b}^{a c d}\right) \omega_{c} \wedge \omega_{d}
\end{aligned}
$$

а также к соотношениям

$$
\begin{aligned}
d B^{a b}{ }_{c}-B^{h b}{ }_{c} \omega_{h}^{a}-B^{a h}{ }_{c} \omega_{h}^{b}+B^{a b}{ }_{h} \omega_{c}^{h} & =B^{a b}{ }_{c h} \omega^{h}+B^{a b}{ }_{c}{ }^{h} \omega_{h}, \\
d B_{a b}{ }^{c}+B_{h b}{ }^{c} \omega_{a}^{h}+B_{a h}{ }^{c} \omega_{b}^{h}-B_{a b}{ }^{h} \omega_{h}^{c} & =B_{a b}{ }^{c h} \omega_{h}+B_{a b}{ }^{c}{ }_{h} \omega^{h}, \\
d B^{a b c}-B^{h b c} \omega_{h}^{a}-B^{a h c} \omega_{h}^{b}-B^{a b h} \omega_{h}^{c} & =B^{a b c}{ }_{h} \omega^{h}+B^{a b c h} \omega_{h}, \\
d B_{a b c}+B_{h b c} \omega_{a}^{h}+B_{a h c} \omega_{b}^{h}-B_{a b h} \omega_{h}^{c} & =B_{a b c}{ }^{h} \omega_{h}+B_{a b c h} \omega^{h},
\end{aligned}
$$

где коэффициенты в правых частях соотношений являются подходящими функциями на пространстве присоединенной $G$-структуры. При этом справедливы формулы

$$
\begin{aligned}
& A_{[b c d]}^{a}=0 \text {, } \\
& \left.\left.A_{b c d}^{a}=B_{b c d}{ }^{a}+B_{b[c}{ }^{a} d\right]+B_{b\left[c^{h}\right.} B_{d] h}{ }^{a}-B_{h b[c} B^{h a} d\right], \\
& A_{b c}^{[a d]}=B_{b c}{ }^{[a d]}+B_{b h}^{[a} B^{d] h}{ }_{c} \text {, }
\end{aligned}
$$

а также формулы, комплексно сопряженные соотношениям (19). Заметим, что из (18) вытекает, что системы функций $\left\{B^{a b}{ }_{c}\right\},\left\{B_{a}{ }^{b c}\right\},\left\{B^{a b c}\right\}$ и $\left\{B_{a b c}\right\}$ на пространстве присоединенной $G$-структуры определяют чистые тензоры на $M$, называемые соответственно виртуальными тензорами первого и второго рода 
и структурными тензорами первого и второго рода [18]. Легко видеть, что сумма виртуальных тензоров обоих родов (умноженная на коэффициент -2) дает вещественный тензор, который мы выше определили как модифицированный виртуальный тензор. То же относится и к структурным тензорам.

Используя (17), (18) и (14), мы точно так же, как и в [18], вычислим компоненты тензора $R$ на пространстве присоединенной $G$-структуры $G_{1}$-многообразия:

$$
\begin{aligned}
R_{a b c d} & =2\left(B_{a b[c d]}+B_{a b}{ }^{h} B_{h c d}\right), \\
R_{\widehat{a} b c d} & =2 A_{b c d}^{a}, \\
R_{\widehat{a} \widehat{b} c d} & =-2\left(B^{a b h} B_{h c d}+B^{a b}{ }_{[c d]}\right), \\
R_{\widehat{a} b c \widehat{d}} & =A_{b c}^{a d}+B^{a d h} B_{h b c}-B^{a h}{ }_{c} B_{h b}{ }^{d},
\end{aligned}
$$

и соотношения, вытекающие из классических свойств симметрии и вещественности тензора $R$.

ОПРЕДЕЛЕНИЕ 3. Почти эрмитово многообразие называется многообразием Вайсмана-Грея (короче, $V G$-многообразием), если оно принадлежит классу $W_{1} \oplus$ $W_{4}$ в классификации Грея-Эрвельи [4].

$V G$-многообразие можно охарактеризовать тождеством

$$
\mu(X, X)=0
$$

С учетом определения тензора $\mu$ запишем эквивалентное тождество:

$$
\nabla_{X}(\Omega)(X, Y)=-\frac{1}{2(m-1)}\left\{\|X\|^{2} \delta \Omega(Y)-\langle X, Y\rangle \delta \Omega(X)+\Omega(X, Y) \delta \Omega(J X)\right\} .
$$

Поляризуя это тождество и покомпонентно расписывая его на пространстве присоединенной $G$-структуры, убеждаемся, что оно равносильно соотношениям

$$
B_{c}^{a b}=\alpha^{[a} \delta_{c}^{b]}, \quad B^{a b c}=B^{[a b c]}
$$

Здесь $\left\{\alpha^{a}, \alpha_{b}\right\}$ - компоненты формы Ли

$$
\alpha=\frac{1}{m-1} \delta \Omega \circ J
$$

Пусть $M-V G$-многообразие. Заметим, что с учетом (21) соотношения $\left(18_{1}\right)$, $\left(18_{2}\right)$ равносильны системе дифференциальных уравнений на компоненты формы Ли:

$$
d \alpha^{a}-\alpha^{b} \omega_{b}^{a}=\alpha^{a}{ }_{b} \omega^{b}+\alpha^{a b} \omega_{b}, \quad d \alpha_{a}+\alpha_{b} \omega_{a}^{b}=\alpha_{a b} \omega^{b}+\alpha_{a}{ }^{b} \omega_{b}
$$

где $\alpha^{a}{ }_{b}=\frac{2}{m-1} B^{a c}{ }_{c b}, \alpha^{a b}=\frac{2}{m-1} B^{a c}{ }_{c}^{b}, \alpha_{a}{ }^{b}=\frac{2}{m-1} B_{a c}{ }^{c b}, \alpha_{a b}=\frac{2}{m-1} B_{a c}{ }^{c} b$. $\mathrm{C}$ другой стороны, так как $\alpha-$ тензор типа $(1,0)$, имеет место соотношение

$$
d \alpha_{i}+\alpha_{j} \omega_{i}^{j}=\alpha_{i, j} \omega^{j}
$$


где $\left\{\alpha_{i, j}\right\}$ - компоненты тензора $\nabla \alpha$. Сравнивая это соотношение с $(22)$, с учетом (14) и линейной независимости базисных форм получаем:

$$
\alpha_{a, b}=\alpha_{a b}-B_{a b}, \quad \alpha_{a}{ }^{b}=\alpha_{a}{ }^{b}+\frac{1}{4}\|\alpha\|^{2} \delta_{a}^{b}-\frac{1}{2} \alpha^{b} \alpha_{a}
$$

и/или комплексно-сопряженные формулы. Здесь $B_{b c}=B_{b c h} \alpha^{h}$ и соответственно $B^{b c}=B^{b c h} \alpha_{h}$.

Проальтернируем $\left(19_{2}\right)$ по нижним индексам с учетом $(21)$ и $\left(19_{1}\right)$ :

$$
B_{b c d}^{a}=-\frac{1}{2} \alpha^{a} B_{b c d}+\frac{1}{2} B_{[b c} \delta_{d]}^{a}+\alpha_{[b c} \delta_{d]}^{a}
$$

Подставляя $(24)$ в $\left(19_{2}\right)$, получаем:

$$
A_{b c d}^{a}=-\frac{1}{6} B_{b[c} \delta_{d]}^{a}+\frac{1}{6} B_{c d} \delta_{b}^{a}-\frac{1}{6} \alpha_{b[c} \delta_{d]}^{a}-\frac{1}{6} \alpha_{[c d]} \delta_{b}^{a}-\frac{1}{3} \alpha_{[c|b|} \delta_{d]}^{a}-\frac{1}{4} \alpha_{b} \alpha_{[c} \delta_{d]}^{a} .
$$

С учетом (20) и (25) получим выражение компонент тензора Риччи $r_{i j}=R^{k}{ }_{i j k}$ на пространстве присоединенной $G$-структуры:

$$
\begin{aligned}
r_{\widehat{a} b}= & 3 B^{a c h} B_{b c h}-A_{b c}^{c a}-\frac{m-1}{4}\left(\frac{1}{2}\|\alpha\|^{2} \delta_{b}^{a}-\alpha^{a} \alpha_{b}\right) \\
& -\frac{1}{2}\left(\alpha_{c}^{c} \delta_{b}^{a}+(m-2) \alpha_{b}^{a}\right), \\
r_{a b}= & -\frac{m-1}{2}\left(\alpha_{a b}+\alpha_{b a}+\alpha_{a} \alpha_{b}\right), \\
r_{\widehat{a} \widehat{b}}= & -\frac{m-1}{2}\left(\alpha^{a b}+\alpha^{b a}+\alpha^{a} \alpha^{b}\right) .
\end{aligned}
$$

ОПРЕДЕЛЕНИЕ 4. Почти эрмитово многообразие называется приближсенно келеровым (короче, $N \mathscr{K}$-многообразием), если оно принадлежит классу $W_{1}$ в классификации Грея-Эрвельи [4] или, что равносильно, на нем выполняется тождество

$$
\nabla_{X}(J) Y=-\nabla_{Y}(J) X
$$

Покомпонентно расписывая это тождество на пространстве присоединенной $G$-структуры, убеждаемся, что оно равносильно соотношениям

$$
B_{c}^{a b}=0, \quad B^{a b c}=B^{[a b c]}
$$

и/или комплексно-сопряженным соотношениям.

Это - один из наиболее интересных и хорошо изученных классов почти эрмитовых многообразий. Замечательные геометрические свойства $N \mathscr{K}$-многообразий привлекают внимание многих исследователей, среди которых следует выделить имя американского геометра Альфреда Грея [19], [20]. 
ОПРЕДЕЛЕНИЕ 5. Почти эрмитово многообразие называется эрмитовым, а его $A \mathscr{H}$-структура интегрируемой, если оно принадлежит классу $W_{3} \oplus W_{4}$ в классификации Грея-Эрвельи [4] или, что равносильно, на нем выполняется тождество

$$
\nabla_{X}(J) Y-\nabla_{J X}(J)(J Y)=0
$$

Хорошо известно [4], что это условие равносильно обращению в нуль тензора Нейенхейса эндоморфизма $J$, а значит, эрмитово многообразие можно рассматривать как комплексное многообразие с фиксированной на нем эрмитовой формой $\langle\langle X, Y\rangle=\langle X, Y\rangle+\sqrt{-1} \Omega(X, Y)$. Покомпонентно расписывая определяющее тождество на пространстве присоединенной $G$-структуры, убеждаемся, что оно равносильно обращению в нуль ее структурных тензоров.

ОПРЕДЕЛЕНИЕ 6. Почти эрмитово многообразие называется келеровым, если на нем вьполняется тождество

$$
\nabla_{X}(J) Y=0
$$

Хорошо известно [4], что это условие равносильно эрмитовости структуры и замкнутости ее фундаментальной формы. Очевидно также, что келеровость $A \mathscr{H}$-структуры равносильна обращению в нуль всех ее структурных и виртуальных тензоров:

$$
B_{c}^{a b}=0, \quad B^{a b c}=0, \quad B_{a b}^{c}=0, \quad B_{a b c}=0
$$

Как уже отмечалось, класс $V G$-структур инвариантен относительно конформных преобразований метрики. При этом $A \mathscr{H}$-структура этого класса локально (глобально) конформна приближенно келеровой тогда и только тогда, когда ее форма Ли замкнута (соответственно точна) [4].

\section{§4. $Z N \mathscr{K}$-многообразия}

Почти эрмитово многообразие назовем конциркулярно приближсено келеровым (короче, $Z N \mathscr{K}$-многообразием), если его метрика (локально) конциркулярна метрике $N \mathscr{K}$-многообразия. Если конциркулярное преобразование метрики $N \mathscr{K}$-многообразия нетривиально, соответствующее $Z N \mathscr{K}$-многообразие назовем собственным.

Из конформной инвариантности класса $V G$-многообразий немедленно вытекает, что всякое $Z N \mathscr{K}$-многообразие автоматически является $V G$-многообразием. Поэтому естественно встает задача выделить в классе $V G$-многообразий подкласс $Z N \mathscr{K}$-многообразий. Для решения этой задачи рассмотрим $V G$-структуру $(J, g)$, локально конформную $N \mathscr{K}$-структуре $\left(J, g^{0}\right)$. Пусть $\sigma$ - определяющая функция соответствуюшего конформного преобразования метрики. Как мы видели, это преобразование будет конциркулярньм тогда и только тогда, когда функция $\sigma$ удовлетворяет уравнению Яно (2). С другой стороны, легко проверить, что тензор аффиннной деформации от римановой связности $\nabla$ метрики $g$ к римановой связности $\nabla^{0}$ метрики $g^{0}$ имеет вид

$$
\nabla_{X}^{0} Y-\nabla_{X} Y=X(\sigma) Y+Y(\sigma) X-\langle X, Y\rangle \operatorname{grad} \sigma
$$


После простых вычислений отсюда вытекает, что если $\Omega^{0}$ - фундаментальная форма соответствующей $N \mathscr{K}$-структуры, то

$$
\delta^{0} \Omega^{0}=\delta \Omega-2(m-1) d \sigma \circ J
$$

Из определения 4 следует, что фундаментальная форма $N \mathscr{K}$-структуры является формой Киллинга, а потому она козамкнута, т.е. $\delta^{0} \Omega^{0}=0$. В частности, если обе структуры приближенно келеровы, то $d \sigma=0$, т.е. $\sigma=$ const. Следовательно, нетривиальное конформное преобразование $N \mathscr{K}$-структуры не может быть $N \mathscr{K}$-структурой. Далее, из $(28)$ следует, что в обшем случае $\delta \Omega=2(m-1) d \sigma \circ J$, откуда

$$
d \sigma=-\frac{1}{2(m-1)} \delta \Omega \circ J=-\frac{1}{2} \alpha
$$

С учетом этого уравнение (2) принимает вид:

$$
\nabla \alpha=-\frac{1}{2} \alpha \otimes \alpha+\beta g, \quad \beta=\beta(\alpha) \in C^{\infty}(M) .
$$

Более того, свертывая это тождество по ковариантным аргументам, получаем, что с необходимостью $\beta=\frac{1}{2 m}\left(\delta \alpha+\frac{1}{2}\|\alpha\|^{2}\right)$.

Обратно, если форма Ли $\alpha V G$-многообразия удовлетворяет этому уравнению, то она замкнута, а значит, и локально точна. Следовательно, если выбрать в соответствуюшей окрестности $U_{p}$ произвольной точки $p \in M$ функцию $\sigma$ такую, что $d \sigma=-\frac{1}{2} \alpha$, то эта функция удовлетворяет уравнению Яно, а значит, является определяющей функцией конциркулярного преобразования метрики. Далее, согласно определению формы Ли $d \sigma=-\frac{1}{2(m-1)} \delta \Omega \circ J$, а значит, $\delta \Omega=2(m-1) d \sigma \circ J$. Из (28) следует, что $\delta^{0} \Omega^{0}=0$, т.е. преобразованная структура имеет козамкнутую фундаментальную форму, а значит, и нулевую форму Ли. Но тогда из (21) и (27) вытекает, что эта структура приближенно келерова. Тем самым доказана

Теорема 3. Многообразие Вайсмана-Грея является $Z N \mathscr{K}$-многообразием тогда и только тогда, когда его форма Ли а удовлетворяет соотношению (29). При этом с необходимостью

$$
\beta=\frac{1}{2 m}\left(\delta \alpha+\frac{1}{2}\|\alpha\|^{2}\right)
$$

СлеДСТВИЕ. $Z N \mathscr{K}$-многообразие не является обобщенным многообразием Xonga. 
ДокАЗАТЕЛЬСтво. Напомним [21], что обобщенным многообразием Хопфа называется локально конформно-келерово многообразие с ненулевой параллельной формой Ли. Поэтому для $Z N \mathscr{K}$-многообразия, являюшегося обобщенным многообразием Хопфа, $\alpha \otimes \alpha=2 \beta g$. Поскольку ранги квадратичных форм справа и слева равны 1 и $2 m=\operatorname{dim} M$ соответственно, равенство допустимо лишш при $\operatorname{dim} M=1$, что невозможно.

Напомним, что А. Греем были введены в рассмотрение три тождества на тензор римановой кривизны почти эрмитовых многообразий, которые он считал ключом к геометрии этих многообразий [22]:

$$
\begin{aligned}
& \text { тождество } R_{1}: \quad\langle R(X, Y) Z, W\rangle=\langle R(J X, J Y) Z, W\rangle ; \\
& \text { тождество } R_{2}: \quad\langle R(X, Y) Z, W\rangle=\langle R(J X, J Y) Z, W\rangle+\langle R(J X, Y) J Z, W\rangle+ \\
& \langle R(J X, Y) Z, J W\rangle ; \\
& \text { тождество } R_{3}: \quad\langle R(X, Y) Z, W\rangle=\langle R(J X, J Y) J Z, J W\rangle .
\end{aligned}
$$

Почти эрмитово многообразие, тензор римановой кривизны которого удовлетворяет тождеству $R_{i}$, он назвал многообразием класса $R_{i}, i=1,2,3$. Там же доказано, что $R_{1} \subset R_{2} \subset R_{3}$. Эти тождества впоследствии изучались многими авторами. Многообразия класса $R_{1}$ назывались паракелеровыми многообразиями [23], а многообразия класса $R_{3}-R K$-многообразиями [24]. Известно, что на келеровых многообразиях удовлетворяются все три тождества, а на приближенно келеровых - тождества $R_{2}$ и $R_{3}$, причем тождество $R_{1}$ удовлетворяется тогда и только тогда, ког да многообразие келерово [22].

Нетрудно проверить, что $A \mathscr{H}$-многообразие $M$ принадлежит классу $R_{3}$ тогда и только тогда, когда $R=0$ или, что равносильно, на пространстве присоединенной $G$-структуры выполняется тождество $R_{\widehat{a} b c d}=0 ; M$ принадлежит классу $R_{2}$ тогда и только тогда, когда $\underset{(0)}{R}=\underset{(7)}{R}=0$ или, что равносильно, на пространстве присоединенной $G$-структуры $R_{\widehat{a} b c d}=R_{a b c d}=0 ; M$ принадлежит классу $R_{1}$ тогда и только тогда, когда $\underset{(0)}{R}=\underset{(7)}{R}=\underset{(1)}{R}=0$ или, что равносильно, на пространстве присоединенной $G$-структуры $R_{\widehat{a} b c d}=R_{a b c d}=R_{\widehat{a} \widehat{b} c d}=0$. Заметим, что из $(20),(21)$ и (25) вытекает

ПРЕДЛОЖЕНИЕ 4. Пусть $M-$ многообразие Вайсмана-Грея. Тогда

1. $\underset{(0)}{R}=0 \Leftrightarrow \alpha_{b c}=-\frac{1}{2} \alpha_{b} \alpha_{c}+B_{b c}$;

2. $\underset{(7)}{R}=0 \Leftrightarrow B_{a b c d}=-\alpha_{[a} B_{b] c d}-\alpha_{(c} B_{d) a b}$;

3. $\underset{(1)}{R}=0 \Leftrightarrow B^{a b h} B_{h d c}=\alpha^{[a}{ }_{[d} \delta_{c]}^{b]}$.

Проводя аналогичные рассуждения для тензора $\mathscr{C}$, с учетом (5) получаем

ПРЕДЛОЖЕНИЕ 5. Пусть $M-$ многообразие Вайсмана-Грея. Тогда

1. $\mathscr{C}=0 \Leftrightarrow \alpha_{[b c]}=B_{b c}$;

2. $\underset{(7)}{\mathscr{C}}=0 \Leftrightarrow B_{a b c d}=-\alpha_{[a} B_{b] c d}-\alpha_{(c} B_{d) a b}$;

3. $\underset{(1)}{\mathscr{C}}=0 \Leftrightarrow B^{a b h} B_{h c d}=\beta^{[a}{ }_{[c} \delta_{d]}^{b]}$, 
әде

$$
\beta_{c}^{a}=\alpha_{c}^{a}+\frac{1}{m-1} r_{c}^{a}-\frac{\kappa}{(2 m-1)(2 m-2)} \delta_{c}^{a} .
$$

СлеДСтвИЕ. Равенство $\underset{(7)}{R}=0$ инвариантно относительно конформных преобразований метрики.

Пусть теперь $M-Z N \mathscr{K}$-многообразие. Заметим, что на пространстве присоединенной $G$-структуры соотношение (29) эквивалентно тождествам

$$
\alpha_{a, b}=-\frac{1}{2} \alpha_{a} \alpha_{b}, \quad \alpha_{a,}{ }^{b}=-\frac{1}{2} \alpha_{a} \alpha^{b}+\beta \delta_{a}^{b}
$$

или с учетом (23)

$$
\begin{aligned}
& \alpha_{a b}=-\frac{1}{2} \alpha_{a} \alpha_{b}+B_{a b}, \\
& \alpha_{a}{ }^{b}=\left(-\frac{1}{4}\|\alpha\|^{2}+\beta\right) \delta_{a}^{b} .
\end{aligned}
$$

Теорема 4. ZN K-многообразие $M$ принадлежст классам $R_{3}$ и $R_{2}$. При этом если оно принадлежит классу $R_{1}$, то оно либо әрмитово, либо является шестимерным $Z N \mathscr{K}$-многообразием с неинтегрируемой структурой.

ДокАЗАТЕльство. Из п. 1 предложения 4 и соотношения $\left(31_{1}\right)$ вытекает, что $R=0$, а значит, $M$ принадлежит классу $R_{3}$. Далее, в [25] показано, что для (0)

$N \mathscr{K}$-многообразий $\underset{(7)}{R}=0$, и в силу следствия предложения 5 это же верно и для $Z N \mathscr{K}$-многообразий. Следовательно, $M$ принадлежит классу $R_{2}$. Наконец, в силу этих фактов и предложения $4 M$ принадлежит классу $R_{1}$ тогда и только тогда, когда $\underset{(1)}{R}=0$, т.е. $B^{a b h} B_{h c d}=\alpha^{[a}{ }_{[c} \delta_{d]}^{b]}$, или с учетом $\left(31_{2}\right)$

$$
B^{a b h} B_{h c d}=\frac{1}{2}\left(\beta-\frac{1}{4}\|\alpha\|^{2}\right) \delta_{c d}^{a b}
$$

где $\delta_{c d}^{a b}=\delta_{c}^{a} \delta_{d}^{b}-\delta_{d}^{a} \delta_{c}^{b}-$ кронекеровская дельта второго порядка.

Коротко напомним [26], что (комплексной) $K$-алгеброй называется тройка $(V,\langle\langle\cdot, \cdot\rangle, *)$, где $V$ - комплексное линейное пространство, $\langle\langle\cdot, \cdot\rangle\rangle$ - положительно определенная эрмитова форма в $V$, “*” - бинарная операция в $V$ (т.е. $\mathbb{R}$-линейное отображение $*: V \times V \rightarrow V)$, обладающая свойствами:

1) $X * Y=-Y * X$

2) $(z X) * Y=X *(z Y)=\bar{z}(X * Y)$,

3) $\langle\langle X * Y, Z\rangle\rangle+\overline{\langle\langle Y, X * Z\rangle\rangle}=0, X, Y, Z \in V, z \in \mathbb{C}$. 
$K$-алгебра $V$ называется абелевой, если $X * Y=0(X, Y \in V)$, и назьвается nростой, если она не содержит нетривиальных идеалов. Рассматривая овеществление $V^{\mathbb{R}}$ пространства $V$, приходим к понятию вещественной формы $K$-алгебры как четверки $\left(V^{\mathbb{R}},\langle\cdot, \cdot\rangle, J, *\right)$, где $\langle\cdot, \cdot\rangle$ - евклидова структура на $V^{\mathbb{R}}$, являющаяся вещественной частью формы $\left\langle\langle\cdot, \cdot\rangle, J\right.$ - комплексная структура на $V^{\mathbb{R}}$, порождающая $V$. Тензор $T$ типа $(2,1)$, задающий операцию “*”, называется композииионным тензором. Очевидно, размерность вешественной формы $K$-алгебры равна удвоенной размерности самой $K$-алгебры. $K$-алгебра называется $K$-алгеброй постоянного типа с, если

$$
\|X * Y\|^{2}=c\|X\|^{2}\|Y\|^{2} \quad(X, Y \in V, \quad\langle X, Y\rangle=0) .
$$

Поляризуя это тождество, несложно получить его полилинейный аналог:

$$
X *(Y * Z)=c(\langle\langle Z, X\rangle\rangle Y-\langle\langle Y, X\rangle\rangle Z), \quad X, Y, Z \in V .
$$

В работе [26] доказано, что класс $K$-алгебр нулевого постоянного типа совпадает с классом абелевых $K$-алгебр, а класс $K$-алгебр ненулевого постоянного типа совпадает с классом трехмерных неабелевых (с необходимостью простых) $K$-алгебр.

Пусть теперь $(M, J, g)-G_{1}$-многообразие. Легко видеть, что в касательном пространстве $T_{m}(M)$ в произвольной точке $m \in M$ канонически индуцируется структура $K$-алгебры $\mathrm{V}_{m}$ с композиционньм тензором $C_{m}$ и эрмитовой метрикой $\left\langle\langle X, Y\rangle=\langle X, Y\rangle+\sqrt{-1} \Omega(X, Y)\right.$. $G_{1}$-многообразие называется многообразием постоянного типа $c$, если эта $K$-алгебра (называемая присоединенной) имеет постоянный тип в каждой его точке. В частности, если $M-Z N \mathscr{K}$-многообразие, непосредственный подсчет с учетом (33) показывает, что условие (32) означает постоянство типа многообразия с постоянной типа $c=\beta-\frac{1}{4}\|\alpha\|^{2}$. Очевидно, абелевость присоединенной $K$-алгебры в каждой точке многообразия, т.е. тождественное равенство нулю структурного тензора $C$, равносильно эрмитовости $Z N \mathscr{K}$-структуры, а ее трехмерность и неабелевость - шестимерности многообразия и неинтегрируемости его $Z N \mathscr{K}$-структуры.

ПРЕДЛОЖЕНИЕ 6. 1) ZN $\mathscr{K}$-многообразие класса $R_{1}$ является әрмитовым тогда и только тогда, когда его форма Ли удовлетворяет соотношению

$$
\delta \alpha=\frac{m-1}{2}\|\alpha\|^{2} .
$$

2) $Z N \mathscr{K}$-многообразие класса $R_{1}$ является шестимерным многообразием с неинтегрируемой структурой тогда и только тогда, когда

$$
\delta \alpha=\|\alpha\|^{2}+b
$$

әде $b \in C^{\infty}(M)$ - ненулевая неотрицательная функиия.

ДокАЗАТЕЛьство. Как мы только что видели, $Z N \mathscr{K}$-многообразие класса $R_{1}$ эрмитово тогда и только тогда, когда его постоянная типа $c=\beta-\frac{1}{4}\|\alpha\|^{2}$ равна нулю, что в силу теоремы 3 равносильно (34). С другой стороны, $Z N \mathscr{K}$-многообразие будет шестимерным и будет иметь неинтегрируемую структуру тог да и только тогда, когда его постоянная типа $c=\beta-\frac{1}{4}\|\alpha\|^{2}$ отлична от нуля, что в силу теоремы 3 и того факта, что в этом случае $m=3$, равносильно $(35)$ при $b=6 c$. 
ПРЕДЛОЖЕНИЕ 7. Компактное $Z N \mathscr{K}$-многообразие $M$ класса $R_{1}$ имеет интегрируемую структуру тогда и только тогда, когда оно келерово. Не существует компактных $Z N \mathscr{K}$-многообразий класса $R_{1}$ с неинтегрируемой структурой.

ДокАЗАТЕЛьство. Очевидно, интегрируемость $Z N \mathscr{K}$-структуры класса $R_{1}$ равносильна равенству нулю его постоянной типа, т.е. соотношению (34). В частности, для такого многообразия $\delta \alpha \geqslant 0$. Но если $M$ компактно, то по теореме Грина $\int_{M} \delta \alpha=0$, что возможно лишш в случае $\delta \alpha=0$. Но тогда в силу $(34)\|\alpha\|^{2}=0$, а значит, $\alpha=0$. Из (21) вытекает, что в этом случае $M-N \mathscr{K}$-многообразие, а поскольку его структура интегрируема, $M$ - келерово многообразие. Аналогично в неинтегрируемом случае с учетом (35) получаем, что $\alpha=0$, а значит, $M-$ некелерово $N \mathscr{K}$-многообразие. Но, как уже упоминалось, такое многообразие не может принадлежать классу $R_{1}$. Следовательно, случай неинтегрируемой структуры невозможен.

СлЕДСТВИЕ. Компактное собственное $Z N \mathscr{K}$-многообразие не может принадлежсать классу $R_{1}$.

ДокАЗАТЕЛЬСТво. В самом деле, если бы оно принадлежало классу $R_{1}$, то по предложению 7 его структура была бы интегрируема. Но тогда она была бы келеровой по тому же предложению, а значит, не была бы собственной.

\section{§5. Некоторые классы $Z N \mathscr{K}$-многообразий}

Почти эрмитово многообразие $M$ называется многообразием постоянного по Ванхекке типа, короче, многообразием $V$-постоянного типа, если на нем выполняется тождество

$$
\begin{gathered}
\langle R(J X, J Y) X, Y\rangle-\langle R(X, Y) X, Y\rangle=c\|X\|^{2}\|Y\|^{2}, \\
X, Y \in \mathfrak{X}(M), \quad\langle X, Y\rangle=\langle X, J Y\rangle=0
\end{gathered}
$$

функция $c \in C^{\infty}(M)$ называется постоянной типа многообразия.

Это понятие было введено Ванхекке [27] как одно из альтернативных обобщений понятия постоянства типа, введенного А. Греем для $N \mathscr{K}$-многообразий [19], и изучалось рядом авторов (см., например, [28]).

Инволюционно поляризуя [15] соотношение (36), с учетом классических свойств симметрии тензора $R$ и невырожденности метрики получаем, что оно равносильно тождествам

$$
\underset{(0)}{R}=0, \quad \underset{(7)}{R}=0, \quad \underset{(1)}{R}(X, Y) Z=\frac{c}{2}\{\langle\langle Y, Z\rangle\rangle X-\langle\langle X, Z\rangle\rangle Y\}
$$

С учетом сделанных выше замечаний получаем следуюший результат.

ТЕОРема 5. Пусть $M-$ почти әрмитово многообразие. Тогда следующие утвержсдения әквивалентны:

1. $M-$ многообразие $V$-постоянного типа с; 
2. $M-$ - АН-многообразие класса $R_{2}$, для которого справедливо тожсдество

$$
\underset{(1)}{R}(X, Y) Z=\frac{c}{2}\{\langle\langle Y, Z\rangle\rangle X-\langle\langle X, Z\rangle\rangle Y\} ;
$$

3. на пространстве присоединенной $G$-структурь

$$
R_{a b c d}=0, \quad R_{\widehat{a} b c d}=0, \quad R_{\widehat{a} \widehat{b} c d}=c \delta_{c d}^{a b} .
$$

С учетом $(20),\left(31_{2}\right)$ и теоремы 5 получаем

ПРЕДЛОЖЕНИЕ 8. ZN $\mathscr{K}$-многообразие является многообразием V-постоянного типа с тогда и только тогда, когда на пространстве присоединенной G-структурьл

$$
B^{a b h} B_{h c d}=\frac{1}{2}\left(\beta-c-\frac{1}{4}\|\alpha\|^{2}\right) \delta_{c d}^{a b} .
$$

ТЕОРема 6. ZN $\mathscr{K}$-многообразие $M$ является многообразием $V$-постоянного типа тогда и только тогда, когда оно либо эрмитово, либо является шестимерным $Z N \mathscr{K}$-многообразием с неинтегрируемой структурой.

ДокАЗАТЕЛЬСтво. Пусть $M$ - многообразие $V$-постоянного типа $c$. Тогда в силу (38) его присоединенная $K$-алгебра имеет постоянный тип $\widetilde{c}=\beta-c-\frac{1}{4}\|\alpha\|^{2}$. Повторяя рассуждение, проведенное при доказательстве теоремы 4 , получаем, что либо $M$ эрмитово, либо оно шестимерно и имеет неинтегрируемую структуру.

Обратно, пусть $Z N \mathscr{K}$-структура на $M$ интегрируема. Тогда ее структурные тензоры - нулевые, а значит, $B^{a b h} B_{h c d}=0$. В силу предложения $8 M$ - многообразие $V$-постоянного типа $c=\beta-\frac{1}{4}\|\alpha\|^{2}$. Если же структура неинтегрируема, а многообразие шестимерно, то его присоединенная $K$-алгебра имеет ненулевой постоянньй тип $\widetilde{c}$, определенный формулой $B^{a b h} B_{h c d}=\frac{\widetilde{c}}{2} \delta_{c d}^{a b}$. Положив $c=\beta-\widetilde{c}-\frac{1}{4}\|\alpha\|^{2}$, получим, что на $M$ выполнено тождество (38), т.е. $M-$ многообразие $V$-постоянного типа $c$.

Изучим свойства секционных кривизн $Z N \mathscr{K}$-многообразий. Прежде всего, из (4) немедленно следует, что секционная кривизна

$$
c(X \wedge Y)=\frac{\langle R(X, Y) X, Y\rangle}{\|X\|^{2}\|Y\|^{2}-\langle X, Y\rangle^{2}}
$$

псевдориманова многообразия $M^{n}$ в двумерном направлении, задаваемом бивектором $X \wedge Y$, связана с его секционной конциркулярной кривизной

$$
c_{Z}(X \wedge Y)=\frac{\langle\mathscr{Z}(X, Y) X, Y\rangle}{\|X\|^{2}\|Y\|^{2}-\langle X, Y\rangle^{2}}
$$

формулой

$$
c_{Z}(X \wedge Y)=c(X \wedge Y)-\frac{\kappa}{n(n-1)} .
$$

$\mathrm{C}$ другой стороны, при конциркулярном преобразовании $g \mapsto \widetilde{g}=e^{2 \sigma} g$ его секционная конциркулярная кривизна, очевидно, меняется по закону

$$
\widetilde{c}_{Z}(X \wedge Y)=e^{-2 \sigma} c_{Z}(X \wedge Y) .
$$

Из (39) и (40) вытекает 
ПРЕДЛОЖЕНИЕ 9. Секиионная кривизна псевдориманова многообразия при конииркулярном преобразовании $g \mapsto \widetilde{g}=e^{2 \sigma} g$ его метрики меняется по закону

$$
\widetilde{c}(X \wedge Y)=e^{-2 \sigma}\left(c(X \wedge Y)-\frac{\kappa}{n(n-1)}\right)+\frac{\widetilde{\kappa}}{n(n-1)},
$$

әде ки $\widetilde{\kappa}-$ скалярные кривизны метрик $g$ и $\widetilde{g}$ соответственно.

СлЕДСТВИЕ. При конииркулярном преобразовании метрика постоянной кривизны переходит в метрику постоянной кривизны.

ДокАзАТЕльство. Это сразу же следует из (41) и того факта, что кривизна $c$ пространства постоянной кривизны связана с его скалярной кривизной $\kappa$ соотношением $\kappa=n(n-1) c$.

Напомним [19], что голоморфной секционной кривизной почти эрмитова многообразия $M$ в направлении вектора $X \in \mathfrak{X}(M)$ назьвается кривизна в двумерном голоморфном направлении $X \wedge J X$. Почти эрмитово многообразие $M$ называется многообразием точечно постоянной голоморфной секционной кривизны с, если

$$
\langle R(X, J X) X, J X\rangle=c\|X\|^{4}, \quad X \in \mathfrak{X}(M), \quad c \in C^{\infty}(M) .
$$

Если $c=$ const, многообразие называется многообразием глобально постоянной голоморфной секиионной кривизны. Дословно повторяя рассуждение, проведенное в [18] при доказательстве теоремы 4, получаем

ПРЕДЛОЖЕНИЕ 10. G1-многообразие является многообразием точечно постоянной голоморфной секиионной кривизнь с тогда и только тогда, когда на пространстве присоединенной G-структуры выполняется соотношение

$$
A_{b c}^{a d}=B_{[b c]}^{a d}+B_{c}^{a h} B_{h b}{ }^{d}-\frac{c}{2} \widetilde{\delta}_{b c}^{a d},
$$

əде $\widetilde{\delta}_{b c}^{a d}=\delta_{b}^{a} \delta_{c}^{d}+\delta_{c}^{a} \delta_{b}^{d}$.

В частности, если $M-Z N \mathscr{K}$-многообразие, в силу (21) и (31) это соотношение принимает вид:

$$
A_{b c}^{a d}=\frac{3\|\alpha\|^{2}-8 \beta}{16} \delta_{b c}^{a d}-\frac{\|\alpha\|^{2}+8 c}{16} \widetilde{\delta}_{b c}^{a d}+\frac{1}{4}\left(\alpha^{a} \alpha_{c} \delta_{b}^{d}+\alpha^{d} \alpha_{b} \delta_{c}^{a}-\alpha^{a} \alpha_{b} \delta_{c}^{d}\right) .
$$

Аналогично с заменой тензора $R$ на тензор $\mathscr{Z}$ определяется постоянство голоморфной конциркулярной кривизны, причем в отличие от свойства глобального постоянства свойство точечного постоянства голоморфной конциркулярной кривизны, как следует из (40), инвариантно относительно конциркулярных преобразований метрики. Далее, из предложения 9 вытекает

ПРЕДЛОЖЕНИЕ 11. Свойство точечного постоянства голоморфной секиионной кривизны почти әрмитова многообразия инвариантно относительно конциркулярных преобразований его метрики. Более точно, при преобразовании метрики $g \mapsto \widetilde{g}=e^{2 \sigma} g$ многообразия постоянной голоморфной секиионной кривизны с эта кривизна меняется по закону

$$
\widetilde{c}=e^{-2 \sigma}\left(c-\frac{\kappa}{n(n-1)}\right)+\frac{\widetilde{\kappa}}{n(n-1)} .
$$


Теорема 7. Келерово многообразие $M$ с невырохсденным әндоморфизмом Риччи не является пространством Фиалкова, а значит, не допускает нетривиальных конииркулярных преобразований метрики.

ДокаЗАтЕльство. Допустим, что $M-$ пространство Фиалкова. Тогда на нем вьполняются соотношения (3), которые можно записать в эквивалентной форме

$$
\tau_{i, j}=\gamma g_{i j}
$$

Условия интегрируемости этой системы дифференциальных уравнений, очевидно, имеют вид:

$$
\tau_{i} R_{j k l}^{i}=\gamma_{, k} g_{j l}-\gamma_{, l} g_{j k}
$$

С учетом (20) и определения келеровой структуры на пространстве присоединенной $G$-структуры эти соотношения примут вид:

$$
\tau_{a} A_{b c}^{a d}=\gamma_{, c} \delta_{b}^{d}
$$

С учетом (19) и определения келеровой структуры $A_{[b c]}^{a d}=0$, а значит, $\gamma_{, c} \delta_{b}^{d}-$ $\gamma, b \delta_{c}^{d}=0$. Свертывая эти соотношения по индексам $b$ и $d$, получаем, что $\gamma, c=0$, $\gamma, \widehat{c}=\overline{\gamma, c}=0$. Следовательно, $d \gamma=0$, т.е. $\gamma=$ const. Более того, из (43) вытекает, что в этом случае $\tau_{a} A_{b c}^{a d}=0$. Свертывая это соотношение по индексам $d$ и $c$, с учетом (26) и определения келеровой структуры получаем, что $r_{j}^{i} \tau_{i}=0$, и ввиду невырожденности эндоморфизма Риччи $\tau=0$, что противоречит определению пространства Фиалкова.

СлЕДСТВИЕ. Комплексное проективное пространство, а также комплексное гиперболическое пространство не являются пространствами Фиалкова.

ДоКАЗАТЕЛЬСТВо немедленно следует из того, что эти пространства Являются пространствами Эйнштейна с ненулевой космологической константой.

Теорема 8. Собственное ZN К-многообразие является многообразием точечно постоянной голоморфной секционной кривизны тогда и только тогда, когда оно с точностью до нетривиального конциркулярного преобразования метрики локально голоморфно изометрично либо комплексному евклидову пространству $\mathbb{C}^{m}$, снабжсенному канонической келеровой структурой, либо шестимерной сфере $S^{6}$, снабженной канонической приближснно келеровой структурой.

ДоКАЗАТЕЛьство. Пусть $M-$ собственное $Z N \mathscr{K}$-многообразие точечно постоянной голоморфной секционной кривизны. Согласно предложению 11 конциркулярное ему $N \mathscr{K}$-многообразие также является многообразием точечно постоянной голоморфной секционной кривизны. Как хорошо известно [25], [29], [30], каждое такое многообразие локально эквивалентно (т.е. локально голоморфно изометрично) одному из следующих многообразий, снабженных канонической келеровой (в последнем случае приближенно келеровой) структурой:

1) комплексному евклидову пространству $\mathbb{C}^{m}$;

2) комплексному проективному пространству $\mathbb{C} P^{m}$; 
3) комплексному гиперболическому пространству $\mathbb{C} D^{m}$;

4) двумерному ориентируемому многообразию $M^{2}$;

5) шестимерной сфере $S^{6}$.

Поскольку любая почти эрмитова структура на $M^{2}$ - келерова, а рассматриваемая структура - собственная, четвертый случай из рассмотрения исключается. Второй и третий случаи исключаются ввиду теоремы 7. Оставшиеся два вида многообразий, а именно $\mathbb{C}^{m}$ и $S^{6}$, являются пространствами постоянной кривизны, а значит, как уже упоминалось, являются пространствами Фиалкова. Мы, однако, приведем прямое доказательство этих фактов, хорошо вписывающееся в контекст наших рассуждений. Именно легко видеть, что условия интегрируемости (42) очевидным образом удовлетворяются на пространстве $\mathbb{C}^{m}$, если выбрать $\gamma=$ const. Таким образом, пространство $\mathbb{C}^{m}$ является пространством Фиалкова. Далее, условия интегрируемости (42) на некелеровом $N \mathscr{K}$-многообразии примут вид

$$
\tau_{a}\left(B^{a d h} B_{h b c}+A_{b c}^{a d}\right)=\gamma, c \delta_{b}^{d}
$$

Как показано в [30], если такое многообразие имеет постоянную голоморфную секционную кривизну $c$, то

$$
B^{a d h} B_{h b c}=\frac{c}{2} \delta_{b c}^{a d}, \quad A_{b c}^{a d}=-\frac{c}{2} \widetilde{d}_{b c}^{a d}
$$

и условия интегрируемости примут вид $\gamma_{, a}=-c \tau_{a}$. Эта система имеет решение относительно $\gamma$ в силу замкнутости формы $\tau$. В силу такого решения условия интегрируемости выполняются, а значит, $S^{6}$ - пространство Фиалкова.

СЛЕДСТВИЕ 1. Собственное $Z N \mathscr{K}$-многообразие является многообразием точечно постоянной голоморфной секционной кривизны тогда и только тогда, когда оно является пространством постоянной кривизны.

ДокАЗАТЕЛЬство. В самом деле, из теоремы 7 следует, что такое многообразие $M$ с точностью до конциркулярного преобразования метрики является пространством постоянной кривизны. Но согласно следствию предложения 9 конциркулярное преобразование переводит метрику постоянной кривизны в метрику постоянной кривизны. Следовательно, $M$ - пространство постоянной кривизны. Обратное очевидно.

СледСтВИЕ 2 (аналог теоремы Шура для $Z N \mathscr{K}$-многообразий). Точечное постоянство голоморфной секиионной кривизны $Z N \mathscr{K}$-многообразия равносильно глобальному постоянству его голоморфной секчионной кривизны.

Этот результат обобщает аналогичное утверждение для $N \mathscr{K}$-многообразий $[29],[30]$.

Теорема 8 дает исчерпывающее описание $Z N \mathscr{K}$-многообразий точечно постоянной голоморфной секционной кривизны и позволяет строить новые интересные примеры дифференциально-геометрических структур. Например, применяя нетривиальное конциркулярное преобразование к метрике сферы $S^{6}$, снабженной канонической приближенно келеровой структурой $(J, g)$, мы получаем новое почти 
эрмитово многообразие $(M, J, \widetilde{g})$. В соответствии со сказанным вьше это многообразие будет собственньм многообразием Вайсмана-Грея с метрикой постоянной кривизны. В силу компактности и односвязности многообразия его кривизна не может быть нулевой или отрищательной. Поэтому с точностью до гомотетии это многообразие изометрично исходному многообразию $\left(S^{6}, g\right)$. Пусть $f: M \rightarrow S^{6}$ - соответствуюшая изометрия. Тогда $\left(f_{*} J, g\right)$ - собственная неинтегрируемая $Z N \mathscr{K}$-структура на $S^{6}$. Аналогично, применяя нетривиальное конциркулярное преобразование метрики к пространству $\mathbb{C}^{m}$, мы получаем собственную интегрируемую $Z N \mathscr{K}$-структуру на полном односвязном многообразии постоянной нулевой либо отрицательной кривизны.

В заключение приведем исчерпывающее описание конформно-плоских собственных $Z N \mathscr{K}$-многообразий.

ТЕОРема 9. Собственное $Z N \mathscr{K}$-многообразие конформно-плоско тогда и только тогда, когда с точностью до нетривиального конциркулярного преобразования метрики оно локально голоморфно изометрично одному из следующих многообразии:

$$
\mathbb{C}^{m}, \quad S_{k}^{6}, \quad k>0, \quad S_{k}^{6} \times S_{-k}^{2}, \quad k>0,
$$

снабжсенных канонической приближсенно келеровой структурой. Здесь символ $S_{k}^{n}$ означает п-мерное пространство постоянной кривизны $k$.

ДокАЗАТЕЛЬСТво. Пусть $M-$ конформно-плоское собственное $Z N \mathscr{K}$-многообразие, $M_{0}-N \mathscr{K}$-многообразие, полученное из $M$ конциркулярным преобразованием метрики. Тогда $M_{0}$ конформно-плоско и в силу [31] локально голоморфно изометрично одному из следующих $N \mathscr{K}$-многообразий:

$$
\text { 1) } \mathbb{C}^{m}, \text { 2) } S_{k}^{2} \times S_{-k}^{2}, k>0, \text { 3) } M^{2}, \text { 4) } S_{k}^{6}, k>0, \text { 5) } S_{k}^{6} \times S_{-k}^{2}, k>0 .
$$

Многообразие типа 3), как и при доказательстве предыдущей теоремы, из рассмотрения автоматически исключается. Многообразие типа 2) является келеровым многообразием с невырожденным тензором Риччи, а значит, в силу теоремы 7 не является пространством Фиалкова. Многообразия типов 1) и 4), как мы видели, являются пространствами Фиалкова, а значит, допускают нетривиальное конциркулярное преобразование метрики. Остается рассмотреть многообразия типа 5). Такие многообразия подробно изучены в [31]. Согласно теореме 4 из [31] для такого многообразия на пространстве присоединенной $G$-структуры справедливы соотношения

$$
B^{a d h} B_{h b c}=t_{[b}^{[a} \delta_{c]}^{d]}, \quad A_{b c}^{a d}=-t_{(b}^{(a} \delta_{c)}^{d)},
$$

где $t$ - подходящий тензор типа $(1,1)$. Не вдаваясь в исследование геометрического смысла этих соотношений, заметим, что с их учетом условия интегрируемости (42), записанные в форме (44), для нашего многообразия примут вид:

$$
\tau_{a}\left(t_{[b}^{[a} \delta_{c]}^{d]}-t_{(b}^{(a} \delta_{c)}^{d)}\right)=\gamma_{, c} \delta_{b}^{d}
$$

или после очевидных упрощений

$$
\gamma_{, c}=-\tau_{a} t_{c}^{a}
$$


Более того, согласно соотношениям из [31] в специализированном $A$-репере для такого многообразия

$$
t_{\beta}^{\alpha}=\frac{1}{3} B \delta_{\beta}^{\alpha}, \quad t_{4}^{4}=-\frac{1}{3} B, \quad t_{\alpha}^{4}=t_{4}^{\alpha}=0,
$$

где $\alpha, \beta=1,2,3, B=B^{a b c} B_{a b c}=$ const. Тогда соотношения (45) примут вид:

$$
\gamma_{, \beta}=-\frac{1}{3} B \tau_{\beta}, \quad \gamma_{, 4}=\frac{1}{3} B \tau_{4}
$$

Эта система имеет решение относительно $\gamma$ в силу замкнутости формы $\tau$. В силу сушествования такого решения условия интегрируемости (42) выполняются, а значит, $M_{0}$ - пространство Фиалкова.

\section{Список литературы}

1. Бессе А. Многообразия Эйнштейна. Т. I, II. М.: Мир, 1990.

2. Vaisman I. On locally and globally conformal Kähler manifold // Trans. Amer. Math. Soc. 1980. V. 262. P. 533-542.

3. Кириченко В. Ф. Локально конформно-келеровы многообразия постоянной голоморфной секционной кривизны // Матем. сб. 1991. Т. 182. №3. С. 354-363.

4. Gray A., Hervella L. M. The sixteen classes of almost Hermitian manifolds and their linear invariants // Ann. Math. Pura Appl. (4). 1980. V. 123. № 4. P. 35-58.

5. Кириченко B. Ф., Щипкова Н. Н. О геометрии многообразий Грея-Вайсмана // УМН. 1994. T. 49. № 2. C. $155-156$.

6. Yano K. Concircular geometry. I-IV // Proc. Imp. Acad. Japan. 1940. V. 16. P. 195-200, 354-360, 442-448, 505-511.

7. Fialkow A. Conformals geodesics // Trans. Amer. Math. Soc. 1939. V. 45. P. 443-473.

8. Синюков $H$. . C. Геодезические отображения римановых пространств. М.: Наука, 1979.

9. Vries H. L. Über Riemannische Räume, die infinitesimal konforme Transformationen gestatten // Math. Z. 1954. V. 60. №3. P. 328-347.

10. Кобаяии ШI., Номидзу K. Основы дифференциальной геометрии. Т. I, II. М.: Наука, 1981.

11. Goldberg S. J., Kobayashi S. The conformal transformation group of compact Riemannian manifold // Amer. J. Math. 1962. V. 84. P. 170-174.

12. Nagano T. The conformal transformations on the space with parallel Ricci tensor // J. Math. Soc. Japan. 1959. V. 11. P. 10-14.

13. Кириченко B. Ф. K-пространства постоянного типа // Сиб. матем. журн. 1976. Т. 17. № 2. C. $282-289$.

14. Кириченко В.Ф. О геометрии однородных $K$-пространств // Матем. заметки. 1981. T. 30. № 4. C. 569-582.

15. Kirichenko V.F. Generalized quasi-Kählerian manifolds and axioms of $C R$-submanifolds in generalized Hermitian geometry. I // Geom. Dedicata. 1994. V. 51. P. 75-104.

16. Кириченко В. $\Phi$. Аксиома $\Phi$-голоморфных плоскостей в контактной метрической геометрии // Изв. АН СССР. Сер. матем. 1984. Т. 48. № 4. С. 711-739.

17. Кириченко В.Ф., Ежсова Н. A. Конформные инварианты многообразий Вайсмана-Грея // УМН. 1996. Т. 51. № 2. С. 163-164.

18. Арсеньева О.Е., Кириченко В.Ф. Автодуальная геометрия обобщенных эрмитовых поверхностей // Матем. сб. 1998. Т. 189. №1. С. 21-44.

19. Gray A. Nearly Kähler manifolds // J. Differential Geom. 1970. V. 4. № 3. P. 283-309.

20. Gray A. The structure of nearly Kähler manifolds // Math. Ann. 1976. V. 223. P. 233-248.

21. Vaisman I. Locally conformal Kähler manifolds with parallel Lie form // Rend. Mat. (6). 1979. V. 12. P. 268-284. 
22. Gray A. Curvature identities for Hermitian and almost Hermitian manifolds // Tôhoku Math. J. (2). 1976. V. 28. № 4. P. 601-612.

23. Rizza J.-B. Varietá parakähleriane // Ann. Mat. Pura Appl. 1974. V. 98. № 4. P. 47-61.

24. Vanhecke L. Almost Hermitian manifolds with $J$-invariant Riemann curvature tensor // Rend. Sem. Mat. Univ. Politec. Torino. 1975-1976. V. 34. P. 487-498.

25. Kirichenko V.F. Generalized quasi-Kählerian manifolds and axioms of $C R$-submanifolds in generalized Hermitian geometry. II // Geom. Dedicata. 1994. V. 52. P. 53-85.

26. Кириченко $B . \Phi . K$-алгебры и $K$-пространства постоянного типа с индефиинитной метрикой // Матем. заметки. 1981. Т. 29. № 2. С. 265-278.

27. Vanhecke L. $\sigma$-hypersurfaces of generalized complex space forms // Hokkaido Math. J. 1977. V. 6. № 1. P. 31-38.

28. Sawaki S., Sekigawa K. Almost Hermitian manifolds with constant holomorphic sectional curvature // J. Differential Geom. 1974. V. 9. P. 123-134.

29. Gray A. Classification des variétes approximativement kähleriennes de courbure sectionelle holomorphe constante // C. R. Acad. Sci. Paris Sér. I Math. 1974. V. 279. № 22. P. 797-800.

30. Кириченко B. Ф. K-пространства постоянной голоморфонй секционной кривизны // Матем. заметки. 1976. Т. 19. № 5. С. 805-814.

31. Игнаточкина Л. А., Кириченко В. Ф. Конформно-инвариантныесвойстваприближенно келеровых многообразий // Матем. заметки. 1999. Т. 66. № 5. С. 653-663.

Московский педагогический

государственный университет
Поступила в редакцию 17.10 .2000 и 16.01 .2002 